DAMTP/96-73

SWAT/132

\title{
DISSIPATING COSMIC VORTONS AND BARYOGENESIS
}

\author{
Anne-Christine Davis ${ }^{1}$ \\ Warren B. Perkins ${ }^{2}$ \\ 1. Department of Applied Mathematics and Theoretical Physics, \\ Silver Street, Cambridge, CB3 9EW, Great Britain \\ 2. Department of Physics, University of Wales Swansea, \\ Singleton Park, Swansea, SA2 8PP
}

\begin{abstract}
Grand unified theories can admit cosmic strings with fermion zero modes which result in the string carrying a current and the formation of stable remnants, vortons. We consider theories in which the zero modes do not survive a subsequent phase transition, for example the electroweak transition, resulting in vorton dissipation. The dissipating vortons can create a baryon asymmetry. We calculate the asymmetry produced, and show that it is maximised if the vortons decay just before they dominate the energy density of the Universe. We further bound the asymmetry produced by the late decay of any relic particle.
\end{abstract}




\section{1) Introduction}

The rich microstructure of cosmic strings is starting to receive considerable attention. In particular, the additional features acquired by the defect core at each subsequent symmetry breaking [1]. The microstruture of the strings has been used to constrain general particle physics theories to ensure that they are consistent with standard cosmology [2]. In particular, if a theory admits cosmic strings which subsequently become superconducting, then an initially weak current on a closed string loop will amplify as the loop contracts. The current may become sufficiently strong to halt the contraction of the loop, preventing it from decaying. A stable state, or vorton [3], is formed. The density of vortons is tightly constrained by the requirement that they do not over close the universe. This has been used in [2] to constrain such models.

Strings can become superconducting due to boson condensates or to fermion zero modes. The resulting vorton is classically stable [4]. The quantum stability is an open question. It has been assumed that, if vortons decay, they do so by quantum mechanical tunnelling. This would result in them being very long lived. However, in the case of fermion superconductivity, the existence of fermion zero modes at high energy does not guarantee that such modes survive subsequent phase transitions. The disappearance of such zero modes could give another channel for the resulting vortons to decay.

For example, one popular particle physics theory which admits cosmic strings is that based on the grand unified group $\mathrm{SO}(10)$. The resulting strings have right handed neutrino, $\nu_{R}$, zero modes. The presence of zero modes means that the string is superconducting, albeit with a neutral current [5]. The current on the string starts as a small random current, resulting from string inter-commuting and self-intersection. If the current is sufficiently strong then vortons will form. However, due to the way the Higg's field couples, the $\nu_{R}$ zero mode ceases to be a zero mode of the theory after the electroweak phase transition[9]. This results in the string current discharging over a finite time. Thus vortons formed before the electroweak phase transition will decay.

Since the underlying theory is a grand unified $\mathrm{SO}(10)$ theory, and the full $\mathrm{SO}(10)$ symmetry is restored in the core of the string, there will be $\mathrm{SO}(10)$ gauge bosons in the string core. When the vorton decays these are released and their out-of-equilibrium decay results in a baryon asymmetry being produced. This is similar to that produced by collapsing string loops [6] or by monopole annihilation [7].

In this letter we address this problem. First we review neutrino currents and the vorton density produced and then calculate the baryogensis resulting from vorton decay and the change in entropy density. For vortons decaying at the electroweak transition the baryon asymmetry produced by this mechanism is not sufficient to account for nucleosynthesis. However, in theories with an intermediate phase transition such a mechanism may give the required baryon asymmetry. We compare our results with a general limit on baryogenesis produced by the late decay of a relic particle.

\section{Fermion Zero Modes and Vortons}

The $\mathrm{SO}(10)$ string has $\nu_{R}$ zero modes [5], the presence of which result in the string being superconducting. Despite being electrically neutral, this still applies to the $\nu_{R}$, the possibility of neutral current carriers in the string was first developed in [8]. The string 
can build up a random current, similar to that in the bosonic case, resulting from string self-intersections and intercommuting. When the string self-intersects or intercommutes there is a finite probability that the fermi levels will be excited. This produces a distortion in the fermi levels, resulting in a current flow, similar to that discussed by Witten [5]. This results in a smaller current than with an external magnetic field, but the current is still sizable for a grand unified string.

For strings that are formed at a temperature $T_{\mathrm{x}}$ and become superconducting at formation, the vorton number density is given by[2]

$$
n_{v}=\nu_{*} f\left(\frac{\beta T_{\mathrm{x}}}{m_{\mathrm{Pl}}}\right)^{\frac{3}{2}} T^{3}
$$

while the vorton mass density is

$$
\rho_{v}=\nu_{*} f\left(\frac{\beta T_{\mathrm{x}}}{m_{\mathrm{Pl}}}\right)^{\frac{5}{4}} T_{\mathrm{x}} T^{3}
$$

where $\nu_{*}, f$ and $\beta$ are factors of order unity.

The neutrino zero modes in $\mathrm{SO}(10)$ GUT do not remain zero modes after the electroweak phase transition [9], becoming instead low lying bound states. While bound states can carry a current, this current is transient[10]. As the current decays, angular momentum is lost, the vortons shrink and eventually decay. The details of the decay process are discussed in ref.11. As the vortons decay the GUT particles they hold are released and then themselves decay. The baryon asymmetry produced by these decaying particles is discussed in the next section.

\section{Baryogenesis from Vortons}

Given the number density of vortons at the electroweak phase transition we can estimate the baryon asymmetry produced by vorton decay using,

$$
\frac{n_{b}}{s}=\frac{n_{v}}{s} \epsilon N
$$

where $s$ is the entropy density, $\epsilon$ is the baryon asymmetry produced by a single $\mathrm{SO}(10)$ GUT particle and $\mathrm{N}$ is the number of GUT particles per vorton. We need to consider two cases: firstly the vortons may decay before they dominate the energy density of the universe and we do not need to know the time scale for vorton decay since $n_{v} / s$ is an invariant quantity. Alternatively, if the vorton energy density does dominate the energy density of the Universe we must modify the temperature evolution of the Universe to allow for entropy generation.

Assuming that the Universe is radiation dominated until after the electroweak phase transition, the temperature of the Universe is simply that of the standard hot big bang. We can estimate the entropy density following vorton decay using the standard result,

$$
s=\frac{2 \pi^{2}}{45} g^{*} T^{3}
$$


where $g^{*}$ is the effective number of degrees of freedom at the electroweak scale $(\simeq 100)$. The vorton to entropy ratio is then

$$
\frac{n_{v}}{s} \simeq\left(\frac{T_{\mathrm{x}}}{m_{\mathrm{Pl}}}\right)^{\frac{3}{2}} \frac{45}{2 \pi^{2} g^{*}} \sim 5 \times 10^{-6}
$$

for $T_{\mathrm{x}} \sim 10^{16} \mathrm{GeV}$.

The number of GUT particles per vorton is given by[2]

$$
N=\left(\frac{\beta T_{\mathrm{x}}}{m_{\mathrm{Pl}}}\right)^{-\frac{1}{4}} \sim 10
$$

and we have

$$
\frac{n_{b}}{s} \sim 10^{-5} \epsilon
$$

Alternatively, the vorton energy density may come to dominate and we must allow for a non-standard temperature evolution. The temperature of vorton-radiation equality, $T_{\text {veq }}$, is given by

$$
T_{\mathrm{veq}}=\frac{\nu_{*} f}{g^{*}}\left(\frac{\beta T_{\mathrm{x}}}{m_{\mathrm{Pl}}}\right)^{\frac{5}{4}} T_{\mathrm{x}} .
$$

If we assume that the vortons decay at some temperature $T_{d}$ and reheat the Universe to a temperature $T_{\mathrm{rh}}$, we have

$$
\hat{g}^{*} T_{\mathrm{rh}}^{4}=\rho_{v}\left(T=T_{\mathrm{d}}\right)=\nu_{*} f\left(\frac{\beta T_{\mathrm{x}}}{m_{\mathrm{Pl}}}\right)^{\frac{5}{4}} T_{\mathrm{x}} T_{\mathrm{d}}^{3},
$$

where $\hat{g}^{*}$ is the number of degrees of freedom for this lower temperature. We then have,

$$
\frac{T_{\mathrm{rh}}}{T_{\mathrm{eq}}}=\left[\frac{g^{*}}{\hat{g}^{*}}\left(\frac{T_{\mathrm{d}}}{T_{\mathrm{eq}}}\right)^{3}\right]^{\frac{1}{4}} \quad \text { or } \quad \frac{T_{\mathrm{rh}}}{T_{\mathrm{d}}}=\left[\frac{g^{*}}{\hat{g}^{*}} \frac{T_{\mathrm{eq}}}{T_{\mathrm{d}}}\right]^{\frac{1}{4}} .
$$

This reheating and entropy generation leads to an extra baryon dilution factor of

$$
\left(\frac{T_{\mathrm{rh}}}{T_{\mathrm{d}}}\right)^{-3}=\left[\frac{g^{*}}{\hat{g}^{*}} \frac{T_{\mathrm{eq}}}{T_{\mathrm{d}}}\right]^{-\frac{3}{4}}
$$

In this case the baryon asymmetry produced by the decaying vortons is given by

$$
\frac{n_{b}}{s}=\frac{n_{v}}{s} N \epsilon\left[\frac{g^{*}}{\hat{g}^{*}} \frac{T_{\mathrm{eq}}}{T_{\mathrm{d}}}\right]^{-\frac{3}{4}}
$$

where the entropy, $s$, is that of the standard big bang model. The Universe now evolves as in the standard big bang model and $n_{b} / s$ remains invariant. Using the above results the asymmetry becomes,

$$
\frac{n_{b}}{s}=\epsilon\left(\nu^{*} f \frac{\hat{g}^{* 3}}{g^{*^{\prime}}}\right)^{\frac{1}{4}} \beta^{\frac{5}{16}}\left(\frac{T_{d}^{12}}{m_{\mathrm{Pl}}^{5} T_{\mathrm{x}}^{7}}\right)^{\frac{1}{16}}
$$


This form is valid if the vortons dominate the energy density of the Universe before they decay, if this is not the case the dilution factor is absent and we have

$$
\frac{n_{b}}{s} \simeq \frac{\epsilon}{g^{*^{\prime}}}\left(\frac{T_{\mathrm{x}}}{m_{\mathrm{Pl}}}\right)^{\frac{5}{4}}
$$

as above.

For a fixed decay temperature, $T_{\mathrm{d}}$, there is a critical formation temperature above which the vortons dominate the energy density of the Universe before they decay. For very high formation temperatures the entropy generated by the decaying vortons washes out the baryon asymmetry, while for low formation temperatures there are few vortons and little baryon asymmetry is created. Although, in this case a sizeable asymmetry can be generated by loop collapse before vortons are formed [6]. The largest baryon asymmetry is produced if the vortons decay just as they come to dominate the energy density of the Universe. This requires a formation temperature,

$$
T_{\mathrm{x}}=\left[\frac{g^{*}}{\nu_{*} f}\left(\frac{m_{\mathrm{Pl}}}{\beta}\right)^{\frac{5}{4}} T_{\mathrm{d}}\right]^{\frac{4}{9}}
$$

and produces the maximal asymmetry given by,

$$
\frac{n_{b}}{s} \sim \frac{\epsilon}{g^{* \frac{4}{9}}}\left(\frac{T_{\mathrm{d}}}{m_{\mathrm{Pl}}}\right)^{\frac{5}{9}}
$$

Taking the most efficient baryon asymmetry generating factor, $\epsilon \sim 0.01$, we find that in order to generate the observed baryon asymmetry we require $T_{\mathrm{d}}>\sim 10^{-13} \mathrm{~m}_{\mathrm{Pl}} \sim 10^{6} \mathrm{GeV}$. Thus vortons decaying at the electroweak scale cannot produce the observed baryon asymmetry, but decaying vortons could generate significant baryon asymmetry if they became unstable at a sufficiently high energy.

We can compare these results with those from a general model of baryogenesis resulting from the delayed decay of a particle. Let the particles have mass $m$. The density of these particles once they have frozen out will be given by

$$
\rho=\alpha T^{3}
$$

where $\alpha$ is a constant assuming there is no entropy generation. For $T \gg m$ the particles are relativistic and, assuming that they are in equilibrium, their number density equals the number density of photons. For $T<m$ the particle number cannot exceed the photon number as we have annihilation processes, but no production processes and we have the bound $\alpha<m$.

If these particles never dominate the energy density, the baryon asymmetry they produce when they decay is not diluted and is simply given by

$$
\frac{n_{b}}{s}=\frac{n}{s} \epsilon \sim \frac{\epsilon \alpha}{g^{*} m}
$$

where $n$ is the number density of these particles and $\epsilon$ is the baryon asymmetry produced per particle. However, the particles will dominate the energy density of the Universe 
if the decay temperature, $T_{\mathrm{d}}$, is less than the temperature of particle-radiation equality, $T_{\text {eq }}=\alpha / g^{*}$. As above we have a reheat temperature, $T_{\mathrm{rh}}=\left(\alpha / g^{*^{\prime}}\right)^{\frac{1}{4}} T_{\mathrm{d}}^{\frac{3}{4}}$, which introduces a dilution factor and gives a diluted baryon asymmetry,

$$
\frac{n_{b}}{s} \sim \frac{\epsilon \alpha}{g^{*} m}\left(\frac{T_{\mathrm{d}}}{T_{\mathrm{rh}}}\right)^{3} \sim \epsilon\left(\frac{\alpha}{g^{*^{\prime}}}\right)^{\frac{1}{4}} \frac{T_{\mathrm{d}}^{\frac{3}{4}}}{m} \leq \frac{\epsilon \alpha}{g^{*} m} .
$$

The equality arises for $T_{\mathrm{d}}=T_{\mathrm{eq}}$.

The main difference between the vorton and particle mechanisms is the presence of the $T_{d} / m_{\mathrm{Pl}}$ suppression factor in the vorton case. The corresponding factor in the particle case is $\alpha / m$ which we can only bound to be less than unity unless we specify the properties of the particle more exactly. In general decaying particles generate baryon asymmetry most efficiently if they decay at any time before they dominate the energy density of the Universe, in contrast to decaying vortons which produce baryon asymmetry most efficiently if they decay just as they come to dominate the energy density. However, this comparison is slightly misleading; we have assumed a fixed decay temperature and have varied the vorton formation temperature to maximise the baryon asymmetry, but the corresponding feature of the particle model, the dependence of $\alpha$ on $m$, is not specified in the general model. The feature that appears both in the vorton model and the general particle model is that entropy generated by the decay of objects that dominate the energy density reduces the net baryon asymmetry produced. In all cases baryon asymmetry is produced most efficiently if the object do not dominate the energy density of the Universe before they decay.

\section{Discussion}

We have shown that remnants of superconducting strings, vortons, can decay after a subsequent phase transition and these dissipating vortons can create baryon asymmetry. In the case of a GUT scale strings decaying at the electroweak scale, the resulting asymmetry is not enough to explain observations. This is due to the fact that vortons dominate the energy density of the Universe long before they decay. Their decay results in a reheating of the Universe and an increase in the entropy density. This reheating is unlikely to have any effect on the standard cosmology following the electroweak phase transition. If however there was an intermediate transition, and the vortons never dominated the energy density of the Universe, then their decay could explain the observed baryon asymmetry of the Universe.

The question should be addressed as to how general the mechanism in this letter is. In many GUT models there is a $\nu_{R}$ which acquires a mass at the grand unified scale. If strings form when the GUT breaks, they may acquire $\nu_{R}$ zero modes. In order to implement the 'see-saw' mechanism the $\nu_{R}$ mixes with the standard neutrinos at the electroweak scale, resulting in a mass matrix with off-diagonal terms. Unless the electroweak Higgs field winds in the region of restored electroweak symmetry $[9,11]$, then the $\nu_{R}$ will cease to be a zero mode after the electroweak phase transition, becoming instead a low-lying bound state. On the other hand, if the electroweak Higgs field winds around the GUT string then the zero mode will remain. In addition, the ordinary quarks and leptons will also become zero modes in the effective electroweak string [12]. 
Neutrino zero modes appear in many GUT theories which produce strings, they can be excited, leading to neutral currents flowing along the strings and consequently to the formation of vortons. Thus vortons will form in a wide class of GUT models and the mechanism we have analysed is not restricted to the specific case of $\mathrm{SO}(10)$ strings.

This work shows that vorton remnants of superconducting strings are not necessarily disastrous because they may not survive from the GUT scale down to nucleosynthesis, even if zero mode bearing vortons turn out to be quantum mechanically stable. The model we have considered still has the Universe radiation dominated at nucleosynthesis and is cosmologically acceptable. Hence the existence or vortons is not enough to rule out a theory, one needs to check that the currents, and hence the vortons, survive subsequent symmetry breakings.

This mechanism could explain the observed baryon asymmetry depending on the scales of the phase transitions leading to vorton formation and vorton dissipation. There is one caveat to this in that any baryon asymmetry created by the dissipating vortons could be erased by sphaleron processes at the electroweak scale. In the case of $\mathrm{SO}(10)$, the asymmetry created is $\mathrm{B}+\mathrm{L}$ preserving, and so is not erased by electroweak processes, which only eliminate a B-L asymmetry. More generally, many grand unified models create a B $+\mathrm{L}$ asymmetry, and so would evade sphaleron processes. The minimal SU(5) theory creates a B-L preserving asymmetry. However, it doesnot have cosmic strings, nor a $\nu_{R}$, so our mechanism is not applicable in this case. Flipped $\mathrm{SU}(5)$ does have embedded defects [13] and a $\nu_{R}$, so our mechanism may apply here if the defects are meta-stable. However, even in the case of flipped $\mathrm{SU}(5)$ the baryon asymmetry produced is not necessarily destroyed by electroweak processes [14]. Some theories with intermediate scale transitions have a $\nu_{R}$, and create a lepton asymmetry. This is then converted to a by sphaleron processes at the electroweak scale [15]. Our analysis would be applicable to such theories.

We have also considered the late decay of any baryon number violating relic and bounded the resulting asymmetry. In our general analysis we have seen that the maximum asymmetry is produced if the relic decays before it dominates the energy density of the Universe. If the decay occurs later than this, reheating and entropy generation occur and the baryon asymmetry is diluted.

This work is supported in part by PPARC and the EU under HCM programme (CHRX-CT94-0423). We wish to thank Stephen Davis for discussions.

\section{References}

[1] W.B Perkins and A.C. Davis, Nucl Phys B406 (1993) 377

[2] R. Brandenberger, B. Carter, A.C. Davis, and M. Trodden, hep-ph/9605382

[3] R. Davis and E.P.S. Shellard, Nucl Phys B323 (1989) 209

[4] B. Carter and X. Martin, Ann Phys 227 (1993) 151; X. Martin and P. Peter, Phys Rev D51 (1995) 4092

[5] E. Witten, Nucl Phys B249 (1985) 557 
[6] R. Brandenberger, A.C. Davis and M. Hindmarsh, Phys Lett B263 (1991) 239

[7] A.C. Davis, M.A. Earnshaw and U.A. Wiedemann, Phys Lett B293 (1992) 123

[8] R.L. Davis, Phys Rev D38 (1988) 3722

[9] A.C. Davis and S.C. Davis, DAMTP/96-72, hep-ph/9608206

[10] C.T. Hill and L.M. Widrow, Phys Lett B189 (1987) 17; M. Hindmarsh, Phys Lett B200 (1988) 429

[11] A.C. Davis, S.C. Davis and W.B. Perkins (in preparation)

[12] A.C. Davis and W.B. Perkins, Phys Lett (in press), DAMTP/96-71, hep-ph/9610292

[13] A.C. Davis and N.F. Lepora, Phys Rev D52 (1995) 7265

[14] J. Ellis, D.V. Nanopoulos, K.A. Olive, Phys Lett B300 (1993) 121

[15] S.A. Abel and K.E.C. Benson, Phys Lett B335 (1994) 179 